\title{
Seasonal Influenza Vaccination: Its Expected and Unexpected Effects
}

\author{
Xu-Sheng Zhang ${ }^{1,2^{*}}$, Richard G Pebody ${ }^{3}$ and John W McCauley ${ }^{4}$ \\ ${ }^{1}$ Statistics, Modelling and Economics Department, Centre for Infectious Disease Surveillance and Control, Public Health England, London, United Kingdom \\ ${ }^{2}$ Medical Research Council Centre for Outbreak Analysis and Modelling, Department of Infectious Disease Epidemiology, Imperial College School of Public Health, \\ London, United Kingdom
}

${ }^{3}$ Respiratory Diseases Department, Centre for Infectious Disease Surveillance and Control, Public Health England, London, United Kingdom

${ }^{4}$ The Francis Crick Institute, Mill Hill Laboratory, The Ridgeway, Mill Hill, London, NW7 1AA, United Kingdom

*Corresponding author: Xu-Sheng Zhang, Statistics, Modelling and Economics Department, Centre for Infectious Disease Surveillance and Control, Public Health England, London, United Kingdom, E-mail: xu-sheng.zhang@phe.gov.uk

Received date: February 29, 2016; Accepted date: March 25, 2016; Published date: March 30, 2016

Copyright: (c) 2016 Xu-Sheng Z, et al. This is an open-access article distributed under the terms of the Creative Commons Attribution License, which permits unrestricted use, distribution, and reproduction in any medium, provided the original author and source are credited.

\begin{abstract}
Vaccination can induce immune response to protect people against antigenically related virus strains. Although influenza is a vaccine preventable infectious disease, seasonal influenza epidemics still occur annually; and occasional but dramatic pandemics emerge. The reason lies in two evolutionary events regarding influenza viruses: antigenic drift and shift. They continuously generate new strains which annual seasonal and pandemic vaccination aims to track. Recently we proposed a mathematical model to examine the interaction between infection and vaccination. The results from our model showed that vaccination for seasonal influenza protects the vaccinated against vaccine strains (its expected effect), however, the effect of seasonal vaccination on the potential emergence of a future pandemic strain (unexpected effect) remains more uncertain. Further, the effectiveness of the proposed universal vaccines that are designed to provide full spectrum immune protection against seasonal and pandemic influenza will depend on their strength of cross-immunity relative to that induced by natural infection.
\end{abstract}

Keywords: Cross-immunity; Mathematical modelling; Pandemic influenza; Seasonal influenza; Vaccination

\section{Short Communication}

Since 1796 when Edward Jenner had shown that a boy, named James Phipps, who was injected with fresh cowpox lesions, gained protection against smallpox, vaccination has become a powerful weapon for human beings to fight against infectious diseases. One of the most pressing and desperate goals is to invent vaccine to control the spread of emerging infectious diseases such as MERS-Cov (Middle East Respiratory Syndrome Corona Virus Infection) within naïve human populations. Although huge efforts have been made to control and eradicate a number of infectious diseases (including Polio, Yaws, Dracunculiaisis and Malaria) that affect human survival and health, only smallpox has been announced as being eliminated from the global human population. Two key vaccination strategies are usually employed to control and prevent infectious diseases: selective vaccination, where the aim is to provide direct protection to the vaccinated groups who are usually at higher risk, and universal programmes, where the aim is to provide both direct protection to targeted groups plus usually indirect protection to the whole population due to herd immunity [1]. To achieve eradication, a number of criteria need to be met - including the absence of a nonhuman reservoir and the availability of an effective intervention usually (though not always), a vaccine. One reason that eradication is not achieved is that vaccination effectiveness and uptake might not be high enough to reach and maintain the herd immunity threshold. Another reason why most infectious diseases are far from being eliminated is the huge and quickly changing biodiversity in infectious agents [2]. When considering eradication of viral infections, the simple structure and small size of virus genomes make them rely on the host to survive and reproduce, and may have the ability to produce different variants through mutation or recombination and reassortment. This leads to a large challenge for using vaccination to protect naïve populations against some viral infections. Influenza virus is one such example.

Seasonal influenza epidemics are caused by influenza A virus and influenza B virus. New strains can be generated through two evolutionary events: antigenic drift due to mutation and antigenic shift generated from reassortment. Mutations in the genome cause small changes in notably the two surface proteins: hemagglutinin and neuraminidase. Antigenic drift slowly creates an increasing variety of strains until one evolves that can infect people who are immune to the pre-existing strains. Due to its ability to escape from immunity, this new strain then replaces older strains as it rapidly sweeps through the human population, resulting in epidemic influenza. However, since the strains produced by drift can still be reasonably similar to the older strains, some people can still be immune to them. Because of this antigenic drift, a particular influenza vaccine usually confers protection for no more than a few years. Therefore, vaccines that are used to control seasonal influenza must be reviewed each year in anticipation of the oncoming winter influenza seasons.

Due to its segmented genome structure, influenza A virus can generate novel strains through reassortment: two strains of influenza A virus when co-infecting the same host cell can exchange their eight gene segments to generate hybrid strains. Historic data indicate that the recent four influenza pandemics are due to reassortment. Laboratory experiments have shown the potentiality of co-infection of human influenza strains (such as $\mathrm{H} 1 \mathrm{~N} 1$ and $\mathrm{H} 3 \mathrm{~N} 2$ ) with avian influenza A viruses (such as $\mathrm{H} 5 \mathrm{~N} 1$ and $\mathrm{H} 9 \mathrm{~N} 2$ ) to generate reassortant strains that are antigenically distinct, and more transmissible and pathogenic than their parental strains $[3,4]$. The novel reassortant 
strains that deviate dramatically in the viral phenotype from their parental strains can have entirely new antigens so that everybody will be susceptible. These strains can spread uncontrollably and potentially cause a pandemic. Our previous study [5] shows that the crossimmunity built from previous exposure to epidemic strains can reduce the probability of the emergence of pandemic influenza viruses via reassortment. To control seasonal and pandemic influenza, there is a strong case to be made for the development of cross-reactive vaccines that induce immunity against a variety of strains [6]. Traditionally, vaccines are designed to target surface proteins hemagglutinin and neuraminidase but, as described above, they have to be reviewed twice annually because the two surface proteins are always changing and specific. In contrast, the inner proteins of the influenza virus are highly stable between influenza A virus strains. Hence, it is suggested that vaccines that target highly conserved inner proteins will elicit crossreactive immune responses and thus heterosubtypic immunity. One focus of pandemic influenza preparedness is to develop so-called universal vaccines, which can generate a wide spectrum of immune response. Therefore the relevant question is: how effective will the current generation of vaccines for seasonal influenza in general and future universal vaccines in particular be in reducing the emergence of pandemic influenza?

Intuitive thinking suggests that vaccination that reduces the activities of endemic strains and consequently the co-infection of different strains - the pre-requirement for reassortment, might constrain the emergence probability of pandemic influenza. However, once a reassortant strain emerges, its potential to sweep through populations and to develop into pandemic influenza depends on the cross-immunity that was induced by vaccination and previous infection. Only when the potential transmissibility of the novel reassortant strains is also reduced following their creation, can the probability of the emergence of pandemic viruses be constrained. Once vaccinated, people can be protected against the natural infection with endemic strains, and sequentially will get rid of the possible crossimmunity created by the natural infection. As traditional vaccines target variable surface proteins while the natural infection spurs immune response from conserved internal proteins, cross-protection induced from vaccination especially with inactivated vaccines is quite likely to be weaker and shorter than those by natural infection, as confirmed by empirical observations [7]. Thus traditional, inactivated vaccines provide protection against endemic strains but at the same time potentially avoid the opportunity of developing strong crossimmunity against other strains [8]. Mathematical models have proven to be useful tools in analysis of the spread and control of infectious diseases and provide valuable insights into infectious disease control strategies [1]. To disentangle the relationships between vaccination and infection, we proposed a mathematical model to investigate this intricate system [7].

We consider a simple situation that a human population that was endemic with one strain of seasonal influenza virus is being challenged by an invader strain. The invader strain could be a distant strain jumping from avian or swine, or from another region. Vaccination has been used to target the endemic strain and may have varying levels of cross-immune protection against the invader strain and any novel reassortant strain that will be generated through reassortment when the endemic strain and the invader strain co-infect. People are also protected if they were previously infected naturally with the endemic strain. Natural infection will also generate cross-immunity protection against invader and reassortant strains.
Numerical simulations show a simple picture: whether vaccination can help reduce the probability of the emergence of pandemic influenza depends on the relationship between cross-immunity induced by vaccination and that by natural infection. When vaccination-induced cross-immunity against invader and reassortant strains are at least of similar strengths to those naturally elicited, vaccination can decrease the emergence probability of pandemic influenza. In addition, repeated vaccination, which may prolong the duration of immunity, will further reduce the probability. Let us consider a situation where natural infection with a seasonal strain generates sufficiently high cross-immunity against a potential pandemic virus strain. Assume that the available vaccine can induce effective protection against seasonal strains (its expected effect) but only relatively weak cross-immunity against the putative pandemic virus strain (its unexpected effect). In an imaginative situation where vaccination was not conducted, the infection-induced cross-immunity could potentially prevent the putative strain from developing into pandemic influenza [5]. With the universal programme of vaccination, the potential strong cross-immunity induced by natural infection would be evaded; with selective vaccination, infection-induced crossimmunity would be avoided in the vaccinated groups (likely of children as the main transmitters of flu). With these two vaccination strategies, the susceptibility of the whole population would be increased because of weak cross-immunity induced by vaccination in relative to that by natural infection. Under this circumstance vaccination is unlikely to decrease the transmissibility of the novel strains and thus to hamper the emergence probability of pandemic influenza. Our results imply that, to reduce the pandemic risk, (universal) vaccines should be designed to have strong cross-immunity against both invader strains and oncoming reassortant strains or at least strong cross-immunity against oncoming novel strains (see Figure 3 of Zhang et al. [7]). The second part of the condition suggests that the conclusion from our investigation is also applicable to the situation where potential pandemic strains directly transfer into human population from other reservoir species such as pigs and birds.

Two types of seasonal influenza vaccines are now available: inactivated and live attenuated vaccines. Both are safe and effective in inducing protective antibody responses against matching seasonal strains, but only live attenuated vaccines, which mimic natural infection, can induce heterosubtypic immunity [9]. This suggests that universal programmes with these vaccines might have different implications as far as pandemic risk might be concerned. Evaluation of the effectiveness of previous seasonal influenza vaccination in preventing $\mathrm{A}(\mathrm{H} 1 \mathrm{~N} 1)$ pdm09 infection has led to varied outcomes: from eliciting partial protection, to offering no protection, to increasing susceptibility to pandemic influenza [10]. In view of our model, different outcomes may result from different exposure histories and different vaccinations in different populations, implying a complicated and variable relationship between immunity induced by natural infection and by vaccination.

As discussed above an ambitious plan for circumventing and controlling pandemic influenza is to generate universal vaccines, which can react and protect the vaccinated to many different strains of influenza viruses. As our model indicates, however, the key is the strength of cross-immunity induced by the universal vaccines. Although putative universal vaccines could provide protection against the whole spectrum of influenza virus strains, low levels of crossimmunity against some potential pandemic strains might result in the failure of the goal of universal vaccines (like small cracks in a very well-packaged wine bottle). Because of the huge antigenic diversity and 
Citation: Zhang XS, Pebody RG, McCauley JW (2016) Seasonal Influenza Vaccination: Its Expected and Unexpected Effects. J Vaccines Vaccin

Page 3 of 3

unpredicted (thus unknown) candidate strains for pandemic influenza, using universal vaccines to simultaneously control both seasonal and pandemic influenza appears to be a very tough task. By surveying the currently circulating strains and carefully selecting vaccine strains, we could secure the expected effect of vaccine against the oncoming seasonal influenza; however, it is difficult to know how seasonal influenza vaccination will affect the probability of the emergence of pandemic influenza viruses. That is, we can successfully target the enemy (i.e., endemic strains) in sight, but we are not sure whether we hamper or help the enemy hidden in forest (i.e., novel strains).

\section{Conclusion}

The huge and fast changing antigenic diversity in influenza virus incurs a big challenge to controlling influenza. Vaccination for seasonal influenza might effectively protect the human population against endemic influenza virus strains but cannot guarantee its effectiveness in constraining the emergence of pandemic influenza.

\section{References}

1. Vynnycky E, White R (2010) An introduction to infectious disease modelling. Oxford University Press.

2. Lipsitch M, O'Hagan RJ (2007) Patterns of antigenic diversity and the mechanisms that maintain them. J Roy Soc Interface 4: 787-802.
3. Li C, Hatta M, Nidom CA, Muramoto Y, Watanabe S, et al. (2010) Reassortment between avian $\mathrm{H} 5 \mathrm{~N} 1$ and human $\mathrm{H} 3 \mathrm{~N} 2$ influenza viruses creates hybrid viruses with substantial virulence. Proc Natl Acad Sci USA 107: 4687-4692.

4. Sun Y, Qin K, Wang JJ, Pu JA, Tang QD, et al. (2011) High genetic compatibility and increased pathogenicity of reassortants derived from avian H9N2 and pandemic H1N1/2009 influenza viruses. Proc Natl Acad Sci USA 108: 4164-4169.

5. Zhang X-S, De Angelis A, White PJ, Charlett A, Pebody RG, et al. (2013) Co-circulation of influenza A virus strains and emergence of pandemic via reassortment: the role of cross-immunity. Epidemics 5: 20-33.

6. Osterhaus A, Fouchier R, Rimmelzwaan G (2011) Towards universal influenza vaccines?. Philos Trans R Soc Lond B Biol Sci 366: 2766-2773.

7. Zhang X-S, Pebody R, De Angelis D, White PJ, Charlett A, et al. (2014) The Possible Impact of Vaccination for Seasonal Influenza on Emergence of Pandemic Influenza via Reassortment. PLoS ONE 9: el14637.

8. Bodewes R, Kreijtz JHCM, Rimmelzwaan G (2009) Yearly influenza vaccinations: a double-edges sword? Lancet Infect Dis 9: 784-788.

9. Hoft DF, Babusis E, Worku S, Spencer CT, Lottenbach K, et al. (2011) Live and inactivated influenza vaccines induce similar humoral responses, but only live vaccines induce diverse T-cell responses in young children. J Infect Dis 204: 845-853.

10. Viboud C, Simonsen L (2010) Does seasonal influenza vaccination increases the risk of illness with the 2009 A/H1N1 pandemic virus?. PLoS Med 7: e1000259. 